\title{
Chemokines in Gestational Diabetes Mellitus
}

\author{
Hongying Liu ${ }^{1}$, Aizhong Liu ${ }^{1,2}$, Atipatsa C. Kaminga ${ }^{1,3}$, Judy McDonald ${ }^{4}$, \\ Shi Wu Wen ${ }^{5,6}$ and Xiongfeng Pan ${ }^{1 *}$
}

\begin{abstract}
${ }^{1}$ Department of Epidemiology and Health Statistics, Xiangya School of Public Health, Central South University, Changsha, China, ${ }^{2}$ Hunan Provincial Key Laboratory of Clinical Epidemiology, Xiangya School of Public Health, Central South University, Changsha, China, ${ }^{3}$ Department of Mathematics and Statistics, Mzuzu University, Mzuzu, Malawi, ${ }^{4}$ McLaughlin Centre for Population Health Risk Assessment, Faculty of Medicine, University of Ottawa, Ottawa, ON, Canada, ${ }^{5}$ OMNI Research Group, Ottawa Hospital Research Institute, Ottawa, ON, Canada, ${ }^{6}$ Department of Obstetrics and Gynaecology and School of Epidemiology and Public Health, University of Ottawa Faculty of Medicine, Ottawa, ON, Canada
\end{abstract}

\section{OPEN ACCESS}

Edited by: Remo Castro Russo, Federal University of Minas Gerais,

Brazil

Reviewed by:

Syeda Sadia Fatima,

Aga Khan University, Pakistan

Gabriele Saccone,

Federico II University Hospital, Italy

Jiamiao Hu,

Fujian Agriculture and Forestry

University, China

Canan YIImaz,

Gazi University, Turkey

${ }^{*}$ Correspondence:

Xiongfeng Pan

p33557@csu.edu.cn

Specialty section: This article was submitted to Cytokines and Soluble Mediators in Immunity,

a section of the journal

Frontiers in Immunology

Received: 06 May 2021 Accepted: 06 January 2022 Published: 08 February 2022

Citation:

Liu H, Liu A, Kaminga AC, McDonald J, Wen SW and Pan X (2022) Chemokines in Gestational Diabetes Mellitus.

Front. Immunol. 13:705852. doi: 10.3389/fimmu.2022.705852
Background: Studies investigating chemokines in gestational diabetes mellitus (GDM) have yielded mixed results. The purpose of this meta-analysis was to explore whether concentrations of chemokines in patients with GDM differed from that of the controls.

Methods: Following Preferred Reporting Items for Systematic Reviews and MetaAnalyses (PRISMA) guidelines, we systematically searched Web of Science, Embase, Cochrane Library, and PubMed databases for articles, published in any language, on chemokines and GDM through August 1st, 2021. The difference in concentrations of chemokines between patients with GDM and controls was determined by a standardized mean difference (SMD) with a 95\% confidence interval (CI), calculated in the meta-analysis of the eligible studies using a random-effects model with restricted maximum-likelihood estimator.

Results: Seventeen studies met the inclusion criteria for the meta-analysis. Altogether, they included nine different chemokines comparisons involving 5,158 participants (1,934 GDM patients and 3,224 controls). Results showed a significant increase of these chemokines (CCL2, CXCL1, CXCL8, CXCL9, and CXCL12) in the GDM patients compared with the controls. However, there was a significant decrease of the chemokines, CCL4, CCL11 and CXCL10, in the GDM patients compared with the controls. Moreover, subgroup analysis revealed a potential role of chemokines as biomarkers in relation to laboratory detection (different sample type and assay methods) and clinical characteristics of GDM patients (ethnicity and body mass index).

Conclusion: GDM is associated with several chemokines (CCL2, CCL4, CCL11, CXCL1, CXCL8, CXCL9, CXCL10 and CXCL12). Therefore, consideration of these chemokines as potential targets or biomarkers in the pathophysiology of GDM development is necessary. Notably, the information of subgroup analysis underscores the importance of exploring putative mechanisms underlying this association, in order to develop new individualized clinical and therapeutic strategies.

Keywords: chemokines, gestational diabetes mellitus, inflammatory, immune microenvironment, meta-analysis 


\section{INTRODUCTION}

In recent years, the incidence of gestational diabetes mellitus (GDM) has increased rapidly worldwide and constitutes a major public health problem (1). GDM leads to adverse short-term perinatal complications (e.g., eclampsia, preeclampsia, placental malfunction, diabetic fetopathy, and jaundice, etc.) and longterm metabolic disorders complications (e.g., increased risk of developing hypertension, Type 2 Diabetes Mellitus (T2DM), metabolic syndrome, and atherosclerotic, etc.) in both the offspring and mothers (2-4).

So far, GDM cannot be cured because its pathogenesis is not well understood (4). Therefore, the emergence of new multidisciplinary treatment approaches is a necessary development. Currently, an increasingly compelling body of research evidence has emerged linking low-grade inflammation state to GDM (5). The immune activation in state of low-grade inflammation is known to decrease $\beta$ cell function and promote insulin resistance in T2DM (6).

Among these immune activation biomarkers, chemotactic cytokines (chemokines) network plays an important role in the pathogenesis of GDM. Preclinical research has classified chemokines into four subfamilies, namely C, CC, CXC, and CX3C, which are identified by position and presence of the cysteine residue (conserved near the $\mathrm{N}$ terminus) (7). Evidence indicates they play an important role in both the immune system and maternal-fetal interface during physiological and pathological pregnancies, which interacts with a group of 7 transmembrane G protein-coupled receptor (GPCRs) $(8,9)$. Specifically, chemokines network is concerned with the interactions among numerous immune activation factors during pregnancy, including delicate chorus between immune coordination and cellular migration, maintenance of the maternal-fetal interface of feto-maternal tolerance, and attraction of immune cells to the sites of ongoing inflammation (9-11). Considering their role in immune coordination and orchestration of the precise spatio-temporal organization of immune cells, chemokines are prime candidates for linking physiological and pathological pregnancies inflammation, and orchestrating pathogenesis of GDM inflammatory crosstalk.

Although the preceding evidence points to a systemic role of the state of chemokines network in the pathology pregnancies inflammation, what is not known is which chemokines are upregulated or down-regulated in GDM and their potential role in the pathogenesis of GDM (11-13). Moreover, it is notoriously difficult to identify a complete nomenclature coverage of chemokines, especially from previous older studies because the chemokines nomenclature has not been uniform (14). To date, more than 50 chemokines acting on 23 discrete receptors have been discovered (15). However, existing studies of chemokines in GDM are frequently underpowered, have mostly disparate methodologies, and often have conflicting state of chemokines (16-18). Therefore, we aimed to select historical and current chemokines potentially involved in the pathogenesis of GDM through extensive retrieval structure terms utilizing as many variant chemokines nomenclatures as possible. Then, we compared the concentrations of the circulating maternal chemokines, and placenta or adipose tissue chemokines between patients with GDM and healthy controls using highquality meta-analytical techniques. We also explored sources of heterogeneity between studies with disparate methodologies, and improved statistical power using subgroup meta-analysis.

\section{METHODS}

\section{Search Strategy and Selection Criteria}

This protocol was registered with the International Prospective Register of Systematic Reviews (PROSPERO: CRD42019148305). In addition, this systematic review and meta-analysis was performed in accordance with the Preferred Reporting Items for Systematic Reviews and Meta-Analyses (PRISMA) guidelines and the Cochrane Handbook (19).

A comprehensive search for articles on chemokines and GDM, published in any language, was conducted systematically on August 1st, 2021 in the following databases: Web of Science, Embase, Cochrane Library, and PubMed. Non-English language studies were translated to English. The complete well-designed search strategy is listed in the Appendix 1. This was designed by professional librarians who made it as comprehensive as possible, while using truncation and wildcards. Boolean operators were also used in the search strategy to allow for variant historical and current names of chemokines.

Two independent reviewers (XP and AK) grouped relevant eligible studies based on title and abstract screening. Inclusion criteria were defined as: (1) subjects met GDM criteria and reported the method for diagnosing GDM; (2) blood, placenta or adipose tissue samples for chemokines measurement were collected from GDM and healthy controls; (3) the mean and standard deviation (SD) of chemokine were reported, or these data could be acquired by contacting the relevant authors. Exclusion criteria were defined as: (1) letters, reviews, interventional clinical trials, case reports or comments; (2) in vitro studies; (3) GDM patients with auto immune and inflammatory disease; and those whose chemokines concentrations were influenced by anti-inflammatory or immunomodulatory drugs for chemokines. Disagreements on eligibility of studies were settled by involving a third reviewer $(\mathrm{AL})$.

\section{Data Extraction}

All eligible studies were stored in a database established by EpiData (version 3.0). Duplicates were removed using EndNote (version X9.1). The following data were extracted from each eligible study by two independent reviewers (SW and AK) using a custom data extraction template. Disagreements about data extraction were settled by consensus. Thus, extracted data included: (1) the title, year of publication and the first author's name; (2) the country; (3) sample size; (4) ethnicity; (5) GDM cases characteristics such as Body Mass Index (BMI), age, systolic blood pressure (SBP), diastolic blood pressure (DBP), fasting plasma glucose (FPG), 2h postprandial blood glucose, insulin, 
insulin resistance index (HOMA-IR), hemoglobin A1c (HbA1c), low-density lipoprotein (LDL), high-density lipoprotein (HDL), cholesterol, and triglycerides; (6) method of chemokine measurement; and (7) sample material of chemokine and mean \pm SD of chemokine concentrations of GDM cases and the comparison group. Furthermore, information about the risk of bias and quality of eligible studies was extracted according to the Newcastle-Ottawa Quality Assessment Scale (NOS) (20).

\section{Statistical Analysis}

To quantitatively summarize the available data, the effect size of chemokines concentrations on GDM for each study was calculated using Cohen's $\mathrm{d}$ as the weighted standardized mean difference (SMD) in chemokines concentrations between GDM cases and the controls (21). Then, the weighted SMDs and their corresponding $95 \%$ confidence intervals (CIs) were pooled in a meta-analysis using a random effects inverse variance model (22, 23). Heterogeneity was assessed using the Cochran's Q statistic test and quantified using the $\mathrm{I}^{2}$ statistic, which measures the proportion of total variability between studies. Thus, the $\mathrm{I}^{2}$ values of $25 \%$ indicate low heterogeneity, $50 \%$ moderate heterogeneity and $75 \%$ high heterogeneity $(24,25)$. Sensitivity analysis was a method that measures how the impact of uncertainties of subgroups can lead to uncertainties on the output variables. It was conducted using the Leave-one-out Method. Subgroup meta-analyses were conducted to explore sources of heterogeneity with respect to the following subgroups based on sample and study characteristics: ethnicity, age, BMI and sample material of chemokine. Furthermore, the between-study probability of publication bias was assessed using the degree of symmetry of funnel plots and the Egger's Linear Regression Test (26). The package, meta, from $\mathrm{R}$ (version 3.5.0) was used for this meta-analysis. The significance level for all statistical tests was set at the 0.05 , and all tests were two-sided.

\section{RESULTS}

\section{Literature Search}

The utilization of the systematic search of four electronic databases yielded a total of 833 studies. After excluding duplicate studies, 722 abstracts were reviewed, of which 539 were excluded. Therefore, the full texts of 183 articles were screened and this led to 17 articles included in this metaanalysis. A flow diagram describing the process of selecting eligible studies is shown in Figure $\mathbf{1}$.

\section{Characteristics of Eligible Studies}

Table 1 summarizes the characteristics and quality of eligible studies. Among the 17 included studies, eleven studies used maternal blood samples, three studies used cord blood samples, seven studies used placenta samples, while three studies used other samples. Also, in terms of the material of chemokines, 13 studies used the protein, while six studies employed the mRNA. Seven studies used Enzyme-Linked Immunosorbent Assay (ELISA) to determine chemokines, six studies employed the polymerase chain reaction (PCR), while four studies used other methods. The average BMI of GDM patients was $28.28 \pm$ 3.83 , and the average age was $31.94 \pm 2.34$. Ten studies were of high quality, whereas seven were of moderate quality, and the NOS scores varied between 5 and 8. Moreover, anthropometric and clinical phenomics characteristics of included studies are described in the Appendix 2 and 3, including SBP, DBP, FPG, $2 \mathrm{~h}$ Post prandial blood glucose, cholesterol, triglycerides, LDL-C, HDL-C, Insulin, HOMA-IR and HbA1c.

Appendix 4 systematically summarizes the classification of chemokines and their receptors that may link chemokines to the pathogenesis of GDM, including forty-six chemokines and eighteen chemokine receptors. In addition, Appendix 5 summarizes the distribution of the cell type of chemokine receptors that may link chemokines to the pathogenesis of GDM in immune microenvironment such as dendric cells, monocytes, macrophages, natural killer cells, Th2 cells, Th17 cells, Treg cells, basophils, CD4 and CD8 T cells.

\section{Main Outcomes}

The eligible studies included nine different chemokines comparisons involving 5,158 participants (1,934 GDM patients and 3,224 controls). The forest plots of specific chemokines are shown in Figure 2. Considering all chemokines involved, chemokines concentrations were significantly higher in the GDM patients than in the controls (SMD=0.87; 95\% CI: 0.41 ; 1.32). However, significantly high heterogeneity was observed in the included studies $\left(I^{2}=98 \%\right)$. Similarly, for CCL2 chemokines reported in five studies that included 523 GDM patients and 523 controls, the concentrations of CCL2 were significantly higher in the GDM patients than in the controls ( $\mathrm{SMD}=1.63$; 95\% CI: 0.72 ; 2.54). Nevertheless, significant heterogeneity was observed in the included studies $\left(I^{2}=97 \%\right)$. For CCL4, the concentrations of CCL4 were significantly lower in the GDM patients than in the controls (SMD $=-3.66 ; 95 \% \mathrm{CI}:-4.30 ;-3.03)$. For CCL11, the concentrations of CCL11 were significantly lower in the GDM patients than in the controls ( $\mathrm{SMD}=-1.26$; 95\% CI: $-1.68 ;-0.83)$. There was no significant difference in the concentrations of CCL3 between the GDM patients and the controls.

Also, for CXC chemokines, CXCL1 concentrations were significantly higher in the GDM patients than in the controls $(\mathrm{SMD}=2.04 ; 95 \% \mathrm{CI}: 1.61 ; 2.48)$; CXCL8 concentrations were significantly higher in the GDM patients than in the controls $(\mathrm{SMD}=1.13 ; 95 \% \mathrm{CI}: 0.32 ; 1.94)$; CXCL9 concentrations were significantly higher in the GDM patients compared with the controls $(\mathrm{SMD}=8.27 ; 95 \% \mathrm{CI}$ : 7.17; 9.36); CXCL10 concentrations were significantly lower in the GDM patients than in the controls (SMD=-1.50; 95\% CI: -2.69; -0.32); CXCL12 concentrations were significantly higher in the GDM patients compared with the controls ( $\mathrm{SMD}=4.38$; 95\% CI: 3.73; 5.04). There was no significant difference between the GDM patients and the controls in the concentrations of CXCL16 and CX3CL1. The Egger's Linear Regression tests and funnel plots revealed low probability of potential publication bias (Figure 3). Sensitivity analysis indicated that any single eligible study influenced little change in the effect size of chemokines on GDM patients, suggesting that the meta-analysis was stable and not driven by any single study. 

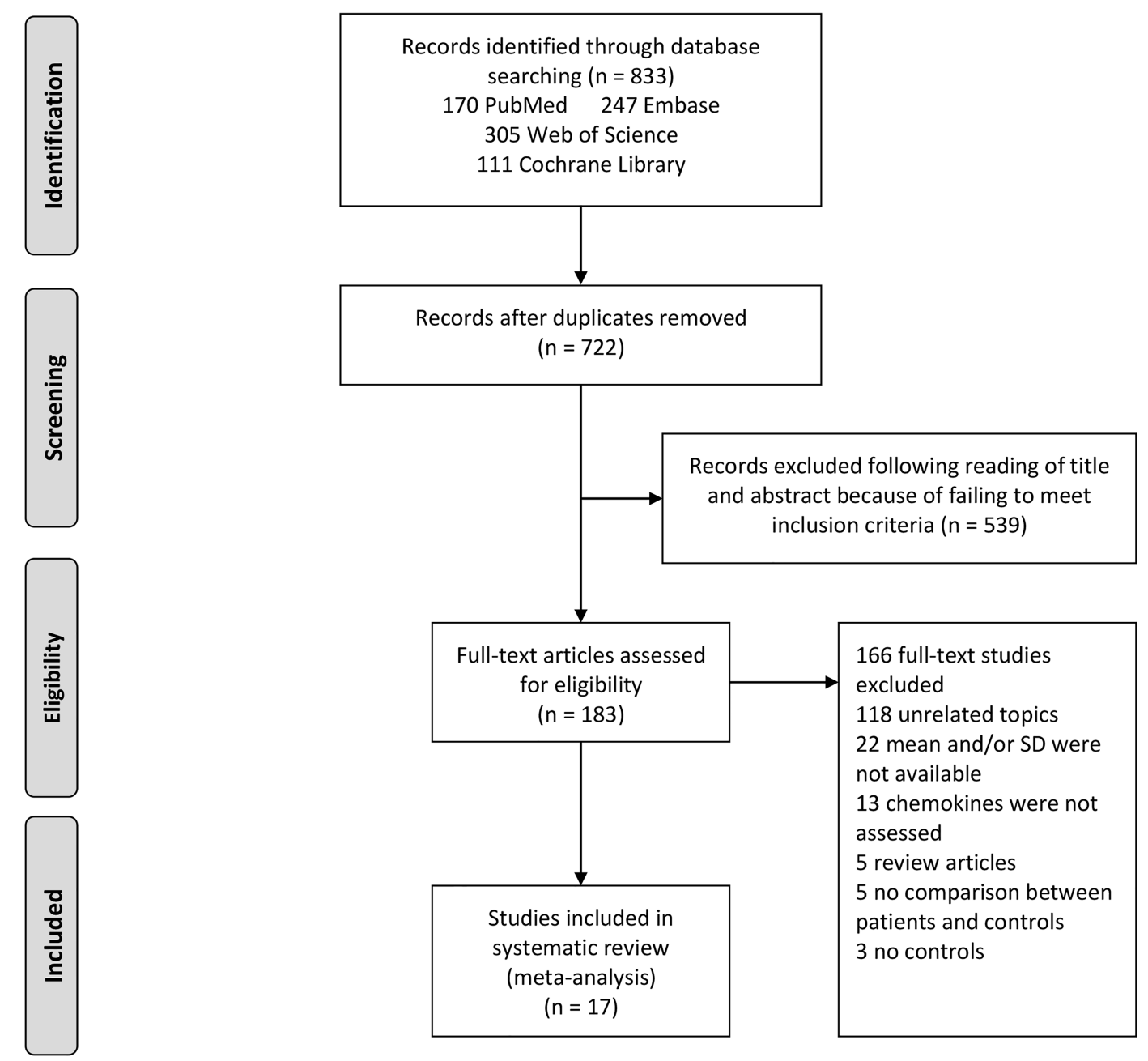

FIGURE 1 | Study selection flow chart. A flow chart demonstrating the selection process of articles included in the analysis as well as in the qualitative summary.

\section{Subgroup Analysis}

Table 2 shows the results of subgroup analysis. Noteworthy, subgroup analysis in this study was divided into laboratory and clinical parts. First, subgroup analyses were conducted to determine the effect of material of chemokines, sample type and assay methods on concentrations of chemokines. These laboratory characteristics were considered for subgroup analyses in view of their well-known effects on chemokines and their pre-clinical implications. Second, subgroup analyses were performed to determine the effect of age, ethnicity, and BMI on concentrations of chemokines. Similarly, these were considered for subgroup analyses following their well-known effects on chemokines and their clinical implications.

Regarding sample of chemokines, only maternal blood chemokines ( $\mathrm{SMD}=1.21 ; 95 \% \mathrm{CI}: 0.63 ; 1.80)$ indicated significantly higher concentrations of chemokines in the GDM patients than in the controls. There was no significant difference in cord blood, placenta or other types of samples. Also, for material type, only protein $(\mathrm{SMD}=0.91 ; 95 \% \mathrm{CI}: 0.37 ; 1.46)$ indicated significantly higher concentrations of chemokines in the GDM patients than in the controls. Studies of mRNA did not reveal a significant difference. Referring to assay methods for chemokines, the ELISA method (SMD=1.45; 95\% CI: 0.79; 2.11). Studies of PCR or other types of methods did not reveal a significant difference.

Considering age of the GDM patients, subgroup metaanalysis showed that concentrations of chemokines were increased significantly in the GDM patients with advanced maternal age $(\mathrm{SMD}=1.41 ; 95 \% \mathrm{CI}: 0.50 ; 2.32)$. Studies of nonadvanced maternal age did not reveal a significant difference. Subgroup meta-analysis according to ethnicity showed that both the Caucasian $(\mathrm{SMD}=0.67 ; 95 \% \mathrm{CI}: 0.07 ; 1.26)$ and the Mongoloid (SMD $=1.45 ; 95 \% \mathrm{CI}: 0.61 ; 2.29$ ) had significantly higher concentrations of chemokines in the GDM patients compared with controls. The effect size in relation to the Mongoloid ethnicity was significantly higher than that of the Caucasian ethnicity. Finally, with regard to BMI, subgroup metaanalysis showed that concentrations of chemokines were 
TABLE 1 | Characteristics of the studies included for the meta-analysis.

\begin{tabular}{|c|c|c|c|c|c|c|c|c|}
\hline Study & & Material & Sample & Country & BMI & Age & Methods & NOS \\
\hline Chueca 2019 & $(27)$ & mRNA & Amnion & Spain & $28.00 \pm 5.03$ & $36.00 \pm 4.06$ & qPCR & 7 \\
\hline Darakhshan 2019 & $(28)$ & Protein & Maternal blood & Iran & $29.70 \pm 1.65$ & $29.60 \pm 1.21$ & ELISA & 6 \\
\hline Ebert 2013 & (29) & Protein & Maternal blood & Germany & $24.50 \pm 6.60$ & $31.00 \pm 7.50$ & ELISA & 8 \\
\hline Hara 2016 & $(17)$ & Protein & Maternal blood/Cord blood/Placenta (villi/extravilli) & Brazil & $28.66 \pm 4.60$ & $29.55 \pm 6.55$ & CBA & 7 \\
\hline Jin 2017 & $(30)$ & mRNA & Placenta/Omental adipose tissues & China & 28.56 & 30.13 & qPCR & 5 \\
\hline Kapustin 2020 & (31) & Protein & Maternal blood & Russia & 28.80 & 34.45 & ELISA & 6 \\
\hline Keckstein 2020 & $(32)$ & Protein & EVT/SCT & Germany & $28.13 \pm 6.96$ & $32.83 \pm 4.56$ & $\mathrm{IHC} / \mathrm{IF}$ & 7 \\
\hline Lappas 2004 & (18) & Protein & Maternal blood & Australia & $25.70 \pm 1.60$ & $35.30 \pm 1.60$ & ELISA & 6 \\
\hline Lekva 2017 & (33) & Protein/mRNA & Maternal blood/PBMC & Norway & $27.80 \pm 5.70$ & $33.10 \pm 3.70$ & EIA/RT-qPCR & 7 \\
\hline Li 2020 & (34) & Protein & Maternal blood/Cord blood & China & $28.31 \pm 4.63$ & $33.59 \pm 5.15$ & UV & 8 \\
\hline Mrizak 2013 & (35) & mRNA & Placenta & Tunisia & $24.90 \pm 2.90$ & 29.50 & qPCR & 8 \\
\hline Murthy 2018 & $(16)$ & Protein & Maternal blood & India & 25.70 & 27.60 & ELISA & 5 \\
\hline Pan 2021 & (36) & mRNA & Placenta & USA & $26.65 \pm 5.73$ & $33.17 \pm 4.65$ & RT-qPCR & 6 \\
\hline Saucedo 2021 & (37) & Protein & Maternal blood & Mexico & 34.80 & 32.00 & $\mathrm{Ml}$ & 7 \\
\hline Stirm 2018 & $(38)$ & Protein/mRNA & Cord blood/Placenta & Germany & $30.60 \pm 5.60$ & $34.00 \pm 4.00$ & Bio-Plex/qPCR & 6 \\
\hline Tang 2021 & (39) & Protein & Maternal blood & China & $21.32 \pm 2.59$ & 32.00 & ELISA & 8 \\
\hline Zhang 2017 & $(40)$ & Protein & Maternal blood/Placenta & China & $38.68 \pm 9.50$ & $29.13 \pm 3.65$ & ELISA & 7 \\
\hline
\end{tabular}

NOS, Newcastle-Ottawa Scale; IHC/IF, immunohistochemistry and immunofluorescence double staining; RT-qPCR, Quantitative reverse transcription polymerase chain reaction; Bio-

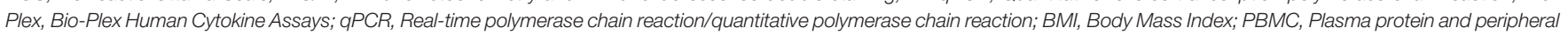
blood mononuclear cells; EIA, Enzyme immunoassay; UV, Ultraviolet spectrophotometry; CBA, Cytometric bead assay; PCR, Polymerase Chain Reaction; MI, Multiplex immunoassay; ELISA, enzyme-linked immunosorbent assay; NR, not report; USA, The United States of America; UK, United Kingdom.

increased in overweight/obese patients with GDM (SMD $=1.41$; 95\% CI: 0.50; 2.32). However, there was no significant difference in the concentrations of chemokines between the nonoverweight/obese GDM patients and the controls.

\section{DISCUSSION}

This is currently the first meta-analysis and systematic review of chemokine ligands and receptors markers in GDM. It found that concentrations of some chemokines were higher in patients with GDM than in controls, while other chemokines had lower concentrations in patients with GDM than in controls. Specifically, the concentrations of the following chemokines were significantly higher in patients with GDM than in controls: CCL2, CXCL1, CXCL8, CXCL9, and CXCL12. On the other hand, the concentrations of the chemokines, CCL4, CCL11 and CXCL10, were significantly lower in patients with GDM than in controls. These results suggest that some chemokines may link immune microenvironment to the pathogenesis of GDM. Therefore, it is imperative to illustrate the unique role of chemokines in the immune microenvironment in order to examine their implications in the pathogenesis, clinical practice and therapeutic targets of GDM.

\section{Pathogenesis Implications}

With regard to the pathogenesis implications, the interactions between chemokines concentrations and maternal immune microenvironment antagonize the release of syncytiotrophoblast debris, which contributes to placental oxidative stress and systemic inflammation (41). Furthermore, the overnutrition and embryomaternal interactions (the balance between immune suppression and tolerance) during pregnancy can be the major causative factor for the process that probably causes and perpetuates a state of lowgrade inflammation, which is common in several pregnancy complications, such as preeclampsia, preterm labor, GDM, and autoimmune diseases (42). These mechanisms of inflammatory responses during pregnancy may link chemokines to the pathogenesis of GDM. In addition, chronic and systemic state of low-grade and sterile inflammation might explain the association between GDM and pathologic state, in which the immune imbalance between pro-inflammatory and anti-inflammatory chemokines has a key role in metabolic abnormalities, glucolipotoxicity, oxidative stress, and tissue-specific insulin resistance (IR) (43).

Specifically, pancreatic islets, peri-pancreatic adipose tissue and immune cells constitute the immune microenvironment of islets. Thus, glucolipotoxicity occurs when the immune microenvironment of islets is exposed to an early damage by genetic or environmental factors, such as overnutrition in the induction of accumulation of elevated levels of glucose/lipids. Consequently, glucolipotoxicity induces sustained activation of various pro-inflammatory and metabolic pathways, and then starts to secrete numerous chemokines $(42,43)$. The effect of chemokines provokes the immune microenvironment of islets in two aspects. First, chemokines and their receptors are decisively involved in interfering with insulin signaling transcriptional mediated molecular and pro-inflammatory pathways. Moreover, chemokines are one of the major causative factors of the activation of the Adenosine 5-Monophosphate-Activated Protein Kinase (AMPK) Pathway. They also lead to the activation of nuclear factor-kappaB $(\mathrm{NF}-\kappa \mathrm{B} / \mathrm{I} \kappa \mathrm{B} \alpha)$ transcriptional mediated molecular, which stimulates a proinflammatory condition and blocks the activation of insulin signaling receptors of $\beta$ cell (44). Meanwhile, chronic exposure to chemokines stimulates the activation of reactive oxygen species/reactive nitrogen species (ROS/RNS) signaling proteins, which is ultimately involved in the endoplasmic reticulum (ER) stress, DNA damage and $\beta$ cell failure (45). 


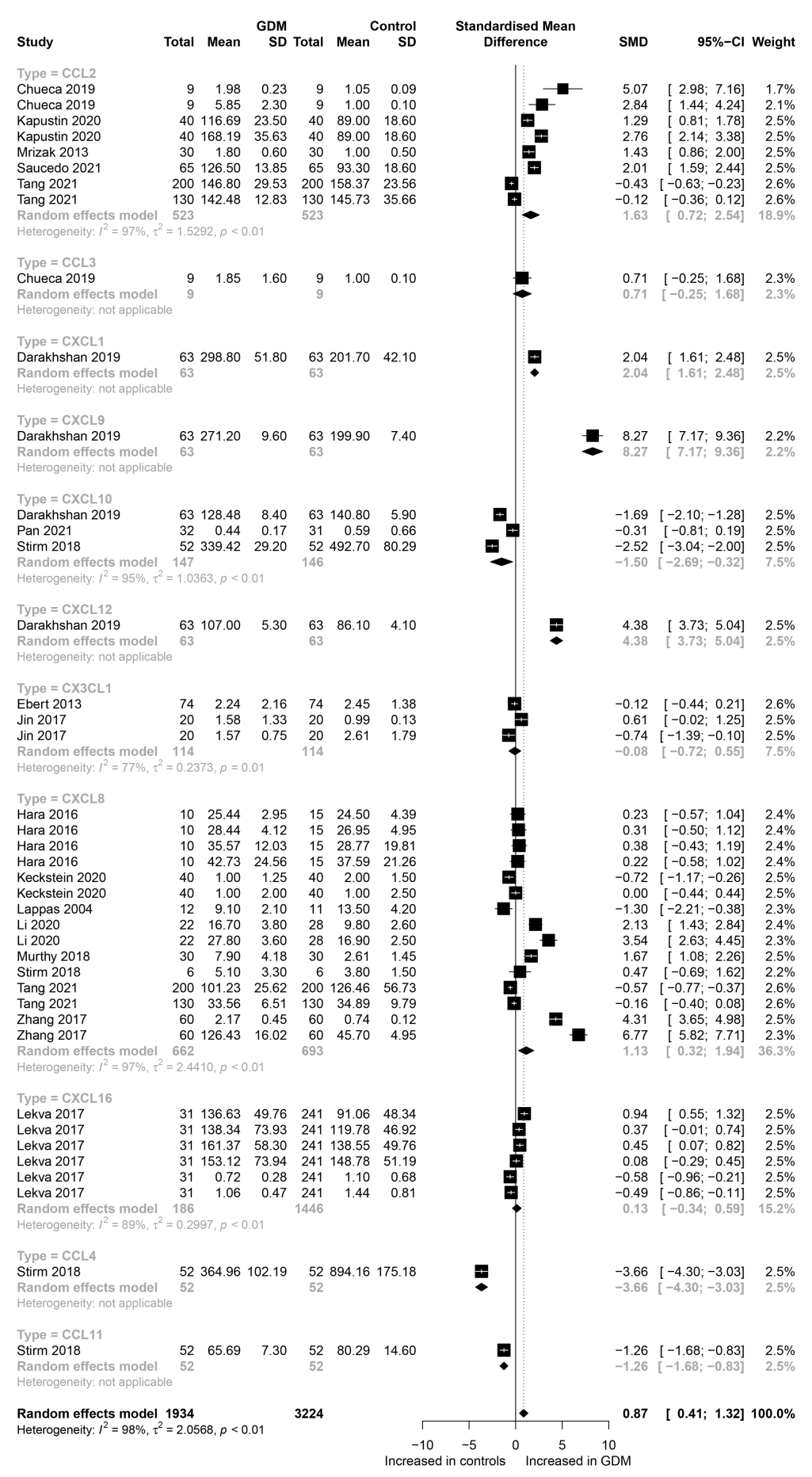

FIGURE 2 | Forest plot of chemokines between GDM patients and controls. Study effect sizes of chemokines differences between GDM and controls. Each data marker represents a study, and the size of the data marker is proportional to the total number of individuals in that study. The summary effect size for each chemokines is denoted by a diamond; GDM, Gestational diabetes mellitus; SMD, standardized mean difference.

Second, chemokines can also lead to the activation of various vasculature or circulating immune cells that enter into the immune microenvironment of islets site (7). A number of these immune cells (i.e., $\mathrm{CD} 8+\mathrm{T}$ cell, $\mathrm{CD} 4+\mathrm{T}$ cell (Th1 cell and
Th17 cell), Natural Killer (NK) cell, eosinophil, macrophage, neutrophil, mast cells, and dendritic cell) are recruited in the immune microenvironment to further release more proinflammatory cytokines (i.e., IL-1 $\beta 、$ IL-6、CRP and IL-1 $\beta$ ), 


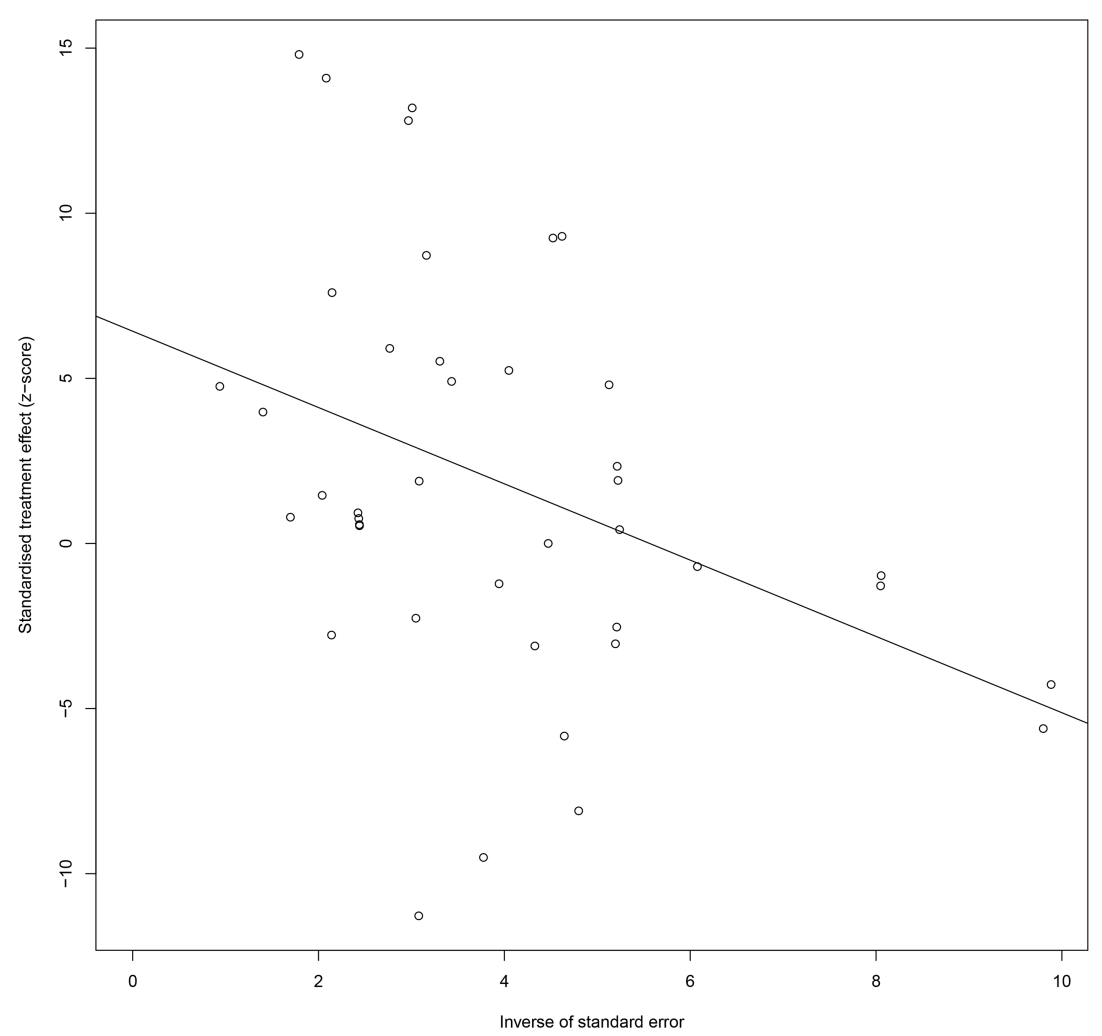

FIGURE 3 | Egger funnel plots of GDM patients compared to controls. GDM patients chemokine and chemokines receptor compared to patients with controls, $t=2.03, p$-value $=0.061$. Egger funnel plots to assess publication bias. Plots show study size as a function of effect size for studies included in the meta-analysis. The dots represent each study. GDM, Gestational diabetes mellitus.

which provoke the pancreatic islet's apoptosis and $\beta$ cell damage by immune attack $(14,15)$. Figure 4 shows the complicated chemokines network in the pancreatic islets' microenvironment of GDM.

\section{Clinical Implications}

The findings of subgroup meta-analysis point to a potential clinical implication in the use of chemokines as biomarkers of GDM. Moreover, the potential role of chemokines as biomarkers

TABLE 2 | Subgroup analysis of chemokine between GDM participants and controls.

\begin{tabular}{|c|c|c|c|c|c|c|}
\hline Subgroup & & SMD & \multicolumn{2}{|c|}{$95 \% \mathrm{Cl}$} & \multicolumn{2}{|c|}{ Heterogeneity } \\
\hline \multirow[t]{2}{*}{ Material } & mRNA & 0.59 & -0.06 & 1.24 & 90.99 & $90.10 \%$ \\
\hline & Protein & 0.91 & 0.37 & 1.46 & 1562.68 & $98.10 \%$ \\
\hline \multirow[t]{3}{*}{ Sample } & Maternal blood & 1.21 & 0.63 & 1.80 & 1015.76 & $98.00 \%$ \\
\hline & Cord blood & -0.74 & -2.66 & 1.17 & 196.45 & $98.00 \%$ \\
\hline & Placenta & 0.96 & -0.14 & 2.05 & 223.31 & $96.40 \%$ \\
\hline & $<30$ & 2.34 & 0.87 & 3.81 & 687.51 & $98.40 \%$ \\
\hline & PCR & 0.59 & -0.06 & 1.24 & 90.99 & $90.10 \%$ \\
\hline & ELISA & 1.45 & 0.79 & 2.11 & 1105.88 & $98.40 \%$ \\
\hline & Other & 0.04 & -1.02 & 1.10 & 436.51 & $97.50 \%$ \\
\hline \multirow[t]{2}{*}{ BMI } & $\geq 28$ & 1.41 & 0.50 & 2.32 & 1393.98 & $98.20 \%$ \\
\hline & $<28$ & 0.05 & -0.23 & 0.34 & 154.46 & $90.90 \%$ \\
\hline
\end{tabular}

Subgroup analyses are performed to compare the concentration of chemokines and chemokines receptors between the GDM and the controls. Heterogeneity was quantified using $P^{2}$ and its significance was tested using the Q statistics. GDM, Gestational diabetes mellitus; NR, not report; SMD, standardized mean difference.

Bold showed significant difference $(P<0.05)$. 


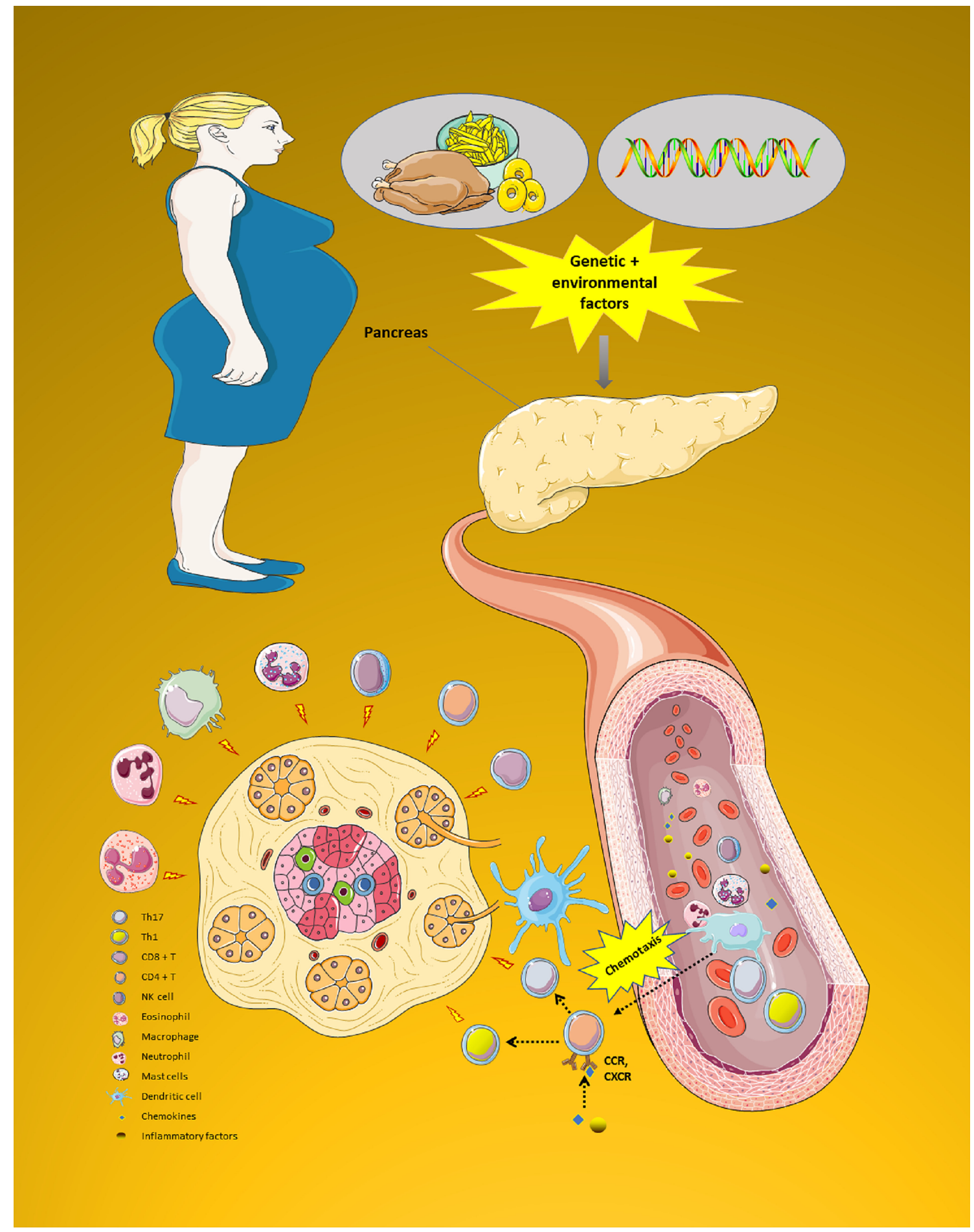

FIGURE 4 | The complicated chemokines network in the pancreatic islets' microenvironment of GDM. The chemokine system plays a variety of roles in the pancreatic islets microenvironment of GDM. First, pancreatic islets are exposed to an early damage by genetic or environmental factors. Then, chemokines can also cause a variety of immune cells to enter the pancreatic islets site to play the role of immune attack. All these processes impact endoplasmic reticulum stress, leading to a reduction in the ability to secrete insulin. Moreover, GDM progression is characterized by progressive secretion of pro-inflammatory chemokines/cytokines caused by $\beta$ cell damage. Due to this process, various immune cell types (i.e., CD8+T cell, CD4+T cell (Th1 cell and Th17 cell), NK cell, eosinophil, macrophage, neutrophil, mast cells, and dendritic cell) are recruited in the pancreatic tissue. These immune cells further release more innate inflammatory cytokines, which contribute to a rapid increase $\beta$ cell death. GDM, Gestational diabetes mellitus; NK, Natural killer. (Drawn by AK). 
is in relation to laboratory detection of biomarkers and clinical characteristics of patients.

In this regard, there were significant differences in the concentrations of chemokines between maternal blood, cord blood and placenta, suggesting that high concentrations of chemokines in maternal circulation cannot enter the fetus through the placental barrier (46). High concentrations of chemokines could be associated with epigenetic changes with DNA methylation including the genes involved in the pathways of immune and inflammatory response, cell growth and death regulation and nervous system development, which increased the risk of future development of T2DM, CAD, obesity, and hypertension in the offspring from mothers with $\operatorname{GDM}(4,47)$.

Furthermore, there were significant differences in the concentrations of chemokines between ELISA, PCR and other types of methods, suggesting that the concentrations of ELISA may have high sensitivity (48). Moreover, considering that a protein is more stable than mRNA, ELISA kit is more convenient in clinical detection (49). Therefore, we recommend that in future clinical and laboratory studies, ELISA samples should be used to detect chemokines in order to obtain more stable results.

Subgroup analysis showed that different ethnicities and ages significantly affect the concentration of chemokines in GDM patients. Subgroup analysis revealed that age significantly affected chemokine circulating concentration in GDM patients. Previous study has shown that the placental functions, including senescence-associated secretory-phenotype production and immune-cell accumulation, gradually decrease in a maternal age-dependent manner, resulting in a higher rate of GDM (50). These results are consistent with age related inflammation kinetics studies, which suggest that patients gradually lose the ability to control excessive inflammatory response with the increase of age. Similarly, previous studies showed that the allele frequencies of single nucleotide polymorphisms of chemokines and their receptor genes were different among different ethnicities (51, 52). Accordingly, this study showed that Mongoloid had a higher effect value than Caucasian. Therefore, we speculate that the genotype-driven treatments in the clinical course of GDM may be a unique window of stratification and individualized pathophysiology-based therapies of GDM patients.

Furthermore, the concentrations of chemokines in this study differed significantly only in GDM patients with $\mathrm{BMI} \geq 28$. This is consistent with previous studies, which suggested that obesity may contribute to the development and progression of GDM. Therefore, it is reasonable to speculate that this may be due to the glucolipotoxicity in obese GDM patients, which further destroys the homeostasis of immune system and chemokines. Further, experimental studies on molecular mechanisms have shown that adipocyte death can activate macrophages and mediate related inflammation and systemic IR (53). Adipocyte death can lead to the formation of peroxynitrite (ONOO-) (54). Among them, ONOO- further induces related inflammation and systemic IR, mediated by oxidative stress products ROS/RNS (55).

\section{Therapeutic Implications}

The development of anti-inflammatory drugs that address placental oxidative stress and systemic state of low-grade inflammation-lowering CCL2, CXCL1, CXCL8, CXCL9 and CXCL10 might be of potential value in GDM treatment. The CCL2 is one of the critical pro-inflammatory chemokines that belongs to the CC chemokine family. Other names of CCL2 include small inducible cytokine A2 (SCYA2), monocyte chemoattractant and activating factor (MCAF) or monocyte chemoattractant protein (MCP)-1. The CCL2 and its cognate receptor CCR2 play a key role in regulating infiltration and migration of Th1 cells, basophil, NK cells, monocytes, and macrophages in the immune microenvironment of islets. Recent research has shown that higher concentrations of CCL2 in serum correlated with early IR, carbohydrate metabolism disorder, obesity development, and preeclampsia development risk (12, 56). CCL2, CCL4, and CCL11 are considered pro-inflammatory chemokines. CCL2 is a CC chemokine with specificity for CCR2 receptors. CCL4 and CCL11 are exerting a wide range of activities through the CCR5 receptors (57). This is consistent with our findings and may suggest that CCL2 is a critical biomarker of GDM as well as a target for therapeutic intervention.

In GDM patients, with the dysfunction of $\beta$ cells, even before $\beta$ cells are widely damaged, CCL4 concentrations may rise ahead of time. While resulting in $\beta$ cells death and early islet graft loss, inflammatory stimuli with a CD40-CD40L interaction could induce the secretion of CCL4 through the Raf/MEK/ERK and NF- $\mathrm{KB}$ pathways in pancreatic islets (58). Therefore, CCL4 concentrations may be caused by the initial inflammatory damage of islet $\beta$ cells.

As a CXC chemokine, the function of CXCL8 is the induction of chemotaxis in its target cells, like neutrophil granulocytes, basophils, T-cells, and adipocytes. There are many receptors capable of binding CXCL8. Those with the most affinity to CXCL8 are receptors CXCR1 and CXCR2 (59). Studies have shown that CXCL8 secreted by adipocytes may be related to complications such as GDM, which are the accumulation of excess accumulation of intra-abdominal fat (60). Increasing evidence suggests that the intra-abdominal fat accumulation is closely related to decreased insulin sensitivity and increased GDM pathophysiology (61). The results of our study suggest that GDM patients have higher concentrations of CXCL8, which is consistent with the findings of previous research. CXCL8 may mediate the downregulation of adiponectin in obesity. Adiponectin can prevent the impairment of insulin signaling, so CXCL8 may plays a crucial and causal role in obesity-linked IR and GDM (62).

Additionally, both the CXCL9 and CXCL10 are the critical pro-inflammatory and angiostasis chemokines that belong to the CXC chemokine family. These chemokines and their cognate receptor CXCR3 play a key role in regulating infiltration and migration of basophils, Th1 cells, CD8+T cells, NK cells, and Treg cells into pancreatic tissue. A clinical study also showed that the concentrations of CXCL10 in T2DM patients were higher than that in the controls (63). Moreover, both CXCL9 and CXCL10 have been attributed to several roles in IR pathogenesis, such as influencing islet $\beta$ cell mass and decreasing $\beta$ cell viability. Similarly, an animal experiment showed that both CXCL9 and CXCL10 played a key role in regulating infiltration and migration of basophils and NK cells into the immune microenvironment of islets, which impaired insulin secretion and function in mice with genetic deletion of 
CXCR3 (64). Besides, CXCL10 leads to the activation of c-Jun Nterminal kinase (JNK), and protein kinase B (Akt) via chemokine receptor CXCR3 $(65,66)$. The CXCL10 also induces the cleavage of p21-activated protein kinase $2(\mathrm{PAK}-2)$ and triggering $\beta$ cell oxidative stress and destruction $(63,66)$. However, this is inconsistent with our findings, possibly due to the small number of studies included in the meta-analyses. Future studies should therefore further explore the relationship between CXCL10 and GDM.

The CXCL1 is one of the critical pro-inflammatory angiogenesis that belongs to the CXC chemokine family. The CXCL1 and its cognate receptor CXCR2 play a key role in regulating infiltration and migration of neutrophils, monocytes, mast cells, basophils, dendric cells, and NK cells in the immune microenvironment of islets. A recent clinical study has also shown higher concentrations of CXCL1 in GDM patients than in controls. Thus, CXCL1 may be involved in the pathogenesis of GDM through endothelial damage and TNF- $\alpha$ production (67). Also, CXCL1 and its cognate receptor CXCR4, and CXCR7 play a key role in lymphopoiesis and promote angiogenesis in the immune microenvironment of islets. Moreover, CXCL12 is one of the critical factors for angiogenesis that belongs to the CXC chemokine family. A sib-pair study also showed that CXCL12 genetic polymorphisms were associated with T2DM (68). Therefore, CXCL12 might probably be involved in the GDM pathophysiologically due to its association with angiogenesis. This is consistent with a previous study, which reported a close relationship between angiogeneic chemokines, such as CXCL1 and CXCL12, and endothelium damage and IR (69).

Furthermore, the CXCL16 is one of the pro-inflammatory single-pass type I membrane protein that belongs to the CXC chemokine family. The CXCL16 and its cognate receptor CXCR6 play a key role in regulating infiltration and migration of Th1 cells, Th17 cells, and NK cells in the immune microenvironment of islets. A clinical study showed elevated concentrations of CXCL16 in T2DM, coronary artery disease (CAD), and GDM in early pregnancy and after 5 years $(70,71)$. Moreover, higher levels of CXCL16 correlated with LDL-C and apoli-poprotein B (apoB) (72). Also, CXCL16 acts as a scavenger receptor on macrophages, hence promoting oxidation and internalization of LDL-C, which may play an important role in the inflammatory response due to lipid accumulation. However, this is inconsistent with our findings, perhaps due to the small number of studies included in the meta-analysis. Future studies need to further explore the relationship between CXCL16 and GDM.

The main strength of this study is that it has summarized the effect of as many chemokines as possible on GDM, which was achieved through a comprehensive search strategy that used multiple variant names of chemokines in a range of databases following PRISMA guidelines. Nevertheless, our analysis used a limited number of studies reporting the effects of the chemokines, CCL3, CCL4, CCL11 and CXCL12, on GDM, which may lead to biased or heterogeneous results. Furthermore, this study was limited by the lack of original research data on confounding variables at the individual level, such as smoking status, alcohol consumption, physical activity, blood pressure, or any combination of these variables. Therefore, these were not taken into account in the meta-analysis. Also, this study used the original data of case-control studies, implying that the findings do not make any causal inference. Therefore, more fundamental mechanistic research and longitudinal study designs, taking into account confounding variables, are essential if we are to truly understand the distinct pathways involved in chemokine biology and the pathophysiological mechanisms of GDM.

\section{CONCLUSIONS}

In this meta-analysis, several chemokines (CCL2, CCL4, CCL11, CXCL1, CXCL8, CXCL9, CXCL10 and CXCL12) were found to be altered in patients with GDM. The contribution of our study was to summarize latest developments in chemokines molecular mechanisms leading to the pathophysiology of GDM development. This allowed future challenges and opportunities for individualized clinical and therapeutic implications of GDM in relation to chemokines to be discussed.

\section{DATA AVAILABILITY STATEMENT}

The original contributions presented in the study are included in the article/Supplementary Material. Further inquiries can be directed to the corresponding author.

\section{AUTHOR CONTRIBUTIONS}

XP and AL contributed to the study design, while SW and AK contributed to the data collection. Statistical analyses and interpretation of results were performed by XP and AK, whereas JM, XP and SW drafted the manuscript and edited the language. All the authors participated in the critical revisions, and approved the final version of the manuscript.

\section{FUNDING}

The research is financially supported by Hunan Provincial Key Laboratory of Clinical Epidemiology and the Hunan Provincial Key Research and Development Program (2018SK2065), China.

\section{ACKNOWLEDGMENTS}

We are grateful to Central South University Library for the assistance during literature search.

\section{SUPPLEMENTARY MATERIAL}

The Supplementary Material for this article can be found online at: https://www.frontiersin.org/articles/10.3389/fimmu.2022.705852/ full\#supplementary-material 


\section{REFERENCES}

1. Szmuilowicz ED, Josefson JL, Metzger BE. Gestational Diabetes Mellitus. Endocrinol Metab Clin North Am (2019) 48(3):479-93. doi: 10.1016/ j.ecl.2019.05.001

2. Rosenn MF. Pregnancy Outcomes in Women With Gestational Diabetes Compared With the General Obstetric Population. Obstet Gynecol (1998) 91 (4):638-9; author reply 639-40. doi: 10.1097/00006250-199804000-00038

3. Lambrinoudaki I, Vlachou SA, Creatsas G. Genetics in Gestational Diabetes Mellitus: Association With Incidence, Severity, Pregnancy Outcome and Response to Treatment. Curr Diabetes Rev (2010) 6(6):393-9. doi: 10.2174/ 157339910793499155

4. Johns EC, Denison FC, Norman JE, Reynolds RM. Gestational Diabetes Mellitus: Mechanisms, Treatment, and Complications. Trends Endocrinol Metab (2018) 29(11):743-54. doi: 10.1016/j.tem.2018.09.004

5. Pantham P, Aye IL, Powell TL. Inflammation in Maternal Obesity and Gestational Diabetes Mellitus. Placenta (2015) 36(7):709-15. doi: 10.1016/ j.placenta.2015.04.006

6. Lontchi-Yimagou E, Sobngwi E, Matsha TE, Kengne AP. Diabetes Mellitus and Inflammation. Curr Diabetes Rep (2013) 13(3):435-44. doi: 10.1007/ s11892-013-0375-y

7. Pan X, Kaminga AC, Wu Wen S, Liu A. Chemokines in Post-Traumatic Stress Disorder: A Network Meta-Analysis. Brain Behav Immun (2021) 92:115-26. doi: 10.1016/j.bbi.2020.11.033

8. Fredriksson R, Lagerström MC, Lundin LG, Schiöth HB. The G-ProteinCoupled Receptors in the Human Genome Form Five Main Families. Phylogenetic Analysis, Paralogon Groups, and Fingerprints. Mol Pharmacol (2003) 63(6):1256-72. doi: 10.1124/mol.63.6.1256

9. Ao D, Li DJ, Li MQ. CXCL12 in Normal and Pathological Pregnancies: A Review. Am J Reprod Immunol (2020) 84(3):e13280. doi: 10.1111/aji.13280

10. Zheng J, Wang H, Zhou W. Modulatory Effects of Trophoblast-Secreted CXCL12 on the Migration and Invasion of Human First-Trimester Decidual Epithelial Cells Are Mediated by CXCR4 Rather Than CXCR7. Reprod Biol Endocrinol (2018) 16(1):17. doi: 10.1186/s12958-018-0333-2

11. Quinn KE, Prosser SZ, Kane KK, Ashley RL. Inhibition of Chemokine (C-X-C Motif) Receptor Four (CXCR4) at the Fetal-Maternal Interface During Early Gestation in Sheep: Alterations in Expression of Chemokines, Angiogenic Factors and Their Receptors. J Anim Sci (2017) 95(3):1144-53. doi: 10.2527/ jas.2016.1271

12. Teler J, Tarnowski M, Safranow K, Maciejewska A, Sawczuk M, Dziedziejko V, et al. CCL2, CCL5, IL4 and IL15 Gene Polymorphisms in Women With Gestational Diabetes Mellitus. Horm Metab Res (2017) 49(1):10-5. doi: 10.1055/s-0042-111436

13. Shi JW, Yang HL, Fan DX, Yang SL, Qiu XM, Wang Y, et al. The Role of CXC Chemokine Ligand 16 in Physiological and Pathological Pregnancies. Am J Reprod Immunol (2020) 83(4):e13223. doi: 10.1111/aji.13223

14. Pan X, Chiwanda Kaminga A, Liu A, Wen SW, Chen J, Luo J. Chemokines in Non-Alcoholic Fatty Liver Disease: A Systematic Review and Network MetaAnalysis. Front Immunol (2020) 11:1802. doi: 10.3389/fimmu.2020.01802

15. Pan X, Kaminga AC, Wen SW, Liu A. Chemokines in Hepatocellular Carcinoma: A Meta-Analysis. Carcinogenesis (2020) 41(12):1682-94. doi: 10.1093/carcin/bgaal06

16. Sudharshana Murthy KA, Bhandiwada A, Chandan SL, Gowda SL, Sindhusree G. Evaluation of Oxidative Stress and Proinflammatory Cytokines in Gestational Diabetes Mellitus and Their Correlation With Pregnancy Outcome. Indian J Endocrinol Metab (2018) 22(1):79-84. doi: 10.4103/ ijem.IJEM_232_16

17. Hara Cde C, Franca EL, Fagundes DL, de Queiroz AA, Rudge MV, HonorioFranca AC, et al. Characterization of Natural Killer Cells and Cytokines in Maternal Placenta and Fetus of Diabetic Mothers. J Immunol Res (2016) 2016:7154524. doi: 10.1155/2016/7154524

18. Lappas M, Permezel M, Rice GE. Release of Proinflammatory Cytokines and 8-Isoprostane From Placenta, Adipose Tissue, and Skeletal Muscle From Normal Pregnant Women and Women With Gestational Diabetes Mellitus. J Clin Endocrinol Metab (2004) 89(11):5627-33. doi: 10.1210/jc.2003-032097

19. Moher D, Liberati A, Tetzlaff J, Altman DG. Preferred Reporting Items for Systematic Reviews and Meta-Analyses: The PRISMA Statement. Bmj (2009) 339:b2535. doi: 10.1136/bmj.b2535
20. Pan X, Wang Z, Wu X, Wen SW, Liu A. Salivary Cortisol in Post-Traumatic Stress Disorder: A Systematic Review and Meta-Analysis. BMC Psychiatry (2018) 18(1):324. doi: 10.1186/s12888-018-1910-9

21. Pan X, Wen SW, Bestman PL, Kaminga AC, Acheampong K, Liu A. Fetuin-A in Metabolic Syndrome: A Systematic Review and Meta-Analysis. PloS One (2020) 15(3):e0229776. doi: 10.1371/journal.pone.0229776

22. Pan X, Kaminga AC, Chen J, Luo M, Luo J. Fetuin-A and Fetuin-B in NonAlcoholic Fatty Liver Disease: A Meta-Analysis and Meta-Regression. Int J Environ Res Public Health (2020) 17(8):2735-51. doi: 10.3390/ijerph17082735

23. Higgins JP, Thompson SG, Deeks JJ, Altman DG. Measuring Inconsistency in Meta-Analyses. Bmj (2003) 327(7414):557-60. doi: 10.1136/bmj.327.7414.557

24. Pan X, Zhao L, Luo J, Li Y, Zhang L, Wu T, et al. Access to Bike Lanes and Childhood Obesity: A Systematic Review and Meta-Analysis. Obes Rev (2021) 22 Suppl 1(Suppl 1):e13042. doi: 10.1111/obr.13042

25. Higgins JP, Thompson SG. Quantifying Heterogeneity in a Meta-Analysis. Stat Med (2002) 21(11):1539-58. doi: 10.1002/sim.1186

26. Egger M, Davey Smith G, Schneider M, Minder C. Bias in Meta-Analysis Detected by a Simple, Graphical Test. Bmj (1997) 315(7109):629-34. doi: 10.1136/bmj.315.7109.629

27. Algaba-Chueca F, Maymo-Masip E, Ejarque M, Ballesteros M, Llaurado G, Lopez C, et al. Gestational Diabetes Impacts Fetal Precursor Cell Responses With Potential Consequences for Offspring. Stem Cells Transl Med (2020) 9 (3):351-63. doi: 10.1002/sctm.19-0242

28. Darakhshan S, Fatehi A, Hassanshahi G, Mahmoodi S, Hashemi MS, Karimabad MN. Serum Concentration of Angiogenic (CXCL1, CXCL12) and Angiostasis (CXCL9, CXCL10) CXC Chemokines Are Differentially Altered in Normal and Gestational Diabetes Mellitus Associated Pregnancies. J Diabetes Metab Disord (2019) 18(2):371-8. doi: 10.1007/ s40200-019-00421-2

29. Ebert T, Hindricks J, Kralisch S, Lossner U, Jessnitzer B, Richter J, et al. Serum Levels of Fractalkine Are Associated With Markers of Insulin Resistance in Gestational Diabetes. Diabetic Med (2014) 31(8):1014-7. doi: 10.1111/ dme. 12451

30. Jin B, Liu L, Zhang S, Cao X, Xu Y, Wang J, et al. Nuclear Magnetic Resonance-Assisted Metabolic Analysis of Plasma for Mild Gestational Diabetes Mellitus Patients. Metab Syndr Relat Disord (2017) 15(9):439-49. doi: $10.1089 /$ met.2017.0065

31. Kapustin RV, Chepanov SV, Babakov VN, Rogovskaya NY, Kopteeva EV, Alekseenkova EN, et al. Maternal Serum Leptin, Adiponectin, Resistin and Monocyte Chemoattractant Protein-1 Levels in Different Types of Diabetes Mellitus. Eur J Obstet Gynecol Reprod Biol (2020) 254:284-91. doi: 10.1016/ j.ejogrb.2020.09.050

32. Keckstein S, Pritz S, Amann N, Meister S, Beyer S, Jegen M, et al. Sex Specific Expression of Interleukin 7, 8 and 15 in Placentas of Women With Gestational Diabetes. Int J Mol Sci (2020) 21(21):8026-41. doi: 10.3390/ijms21218026

33. Lekva T, Michelsen AE, Aukrust P, Paasche Roland MC, Henriksen T, Bollerslev J, et al. CXC Chemokine Ligand 16 Is Increased in Gestational Diabetes Mellitus and Preeclampsia and Associated With Lipoproteins in Gestational Diabetes Mellitus at 5 Years Follow-Up. Diabetes Vasc Dis Res (2017) 14(6):525-33. doi: 10.1177/1479164117728011

34. Li YX, Long DL, Liu J, Qiu D, Wang J, Cheng X, et al. Gestational Diabetes Mellitus in Women Increased the Risk of Neonatal Infection via Inflammation and Autophagy in the Placenta. Med (Baltimore) (2020) 99(40):e22152. doi: 10.1097/MD.0000000000022152

35. Mrizak I, Grissa O, Henault B, Fekih M, Bouslema A, Boumaiza I, et al. Placental Infiltration of Inflammatory Markers in Gestational Diabetic Women. Gen Physiol Biophys (2014) 33(2):169-76. doi: 10.4149/gpb_2013075

36. Pan X, Jin X, Wang J, Hu Q, Dai B. Placenta Inflammation Is Closely Associated With Gestational Diabetes Mellitus. Am J Transl Res (2021) 13 (5):4068-79.

37. Saucedo R, Valencia J, Moreno-Gonzalez LE, Pena-Cano MI, ArandaMartinez A, Garcia Y, et al. Maternal Serum Adipokines and Inflammatory Markers at Late Gestation and Newborn Weight in Mothers With and Without Gestational Diabetes Mellitus. Ginekol Pol (2021) 10.5603/ GP.a2021.0083. doi: 10.5603/GP.a2021.0083

38. Stirm L, Kovarova M, Perschbacher S, Michlmaier R, Fritsche L, Siegel-Axel D, et al. BMI-Independent Effects of Gestational Diabetes on Human Placenta. J Clin Endocrinol Metab (2018) 103(9):3299-309. doi: 10.1210/jc.2018-00397 
39. Tang M, Luo M, Lu W, Zhang R, Liang W, Gu J, et al. Nerve Growth Factor is Closely Related to Glucose Metabolism, Insulin Sensitivity and Insulin Secretion in the Second Trimester: A Case-Control Study in Chinese. Nutr Metab (Lond) (2020) 17(1):98. doi: 10.1186/s12986-020-00523-2

40. Zhang J, Chi H, Xiao H, Tian X, Wang Y, Yun X, et al. Interleukin 6 (IL-6) and Tumor Necrosis Factor Alpha (TNF-Alpha) Single Nucleotide Polymorphisms (SNPs), Inflammation and Metabolism in Gestational Diabetes Mellitus in Inner Mongolia. Med Sci Monit (2017) 23:4149-57. doi: $10.12659 / \mathrm{msm} .903565$

41. Lekva T, Norwitz ER, Aukrust P, Ueland T. Impact of Systemic Inflammation on the Progression of Gestational Diabetes Mellitus. Curr Diabetes Rep (2016) 16(4):26. doi: 10.1007/s11892-016-0715-9

42. Kalagiri RR, Carder T, Choudhury S, Vora N, Ballard AR, Govande V, et al. Inflammation in Complicated Pregnancy and Its Outcome. Am J Perinatol (2016) 33(14):1337-56. doi: 10.1055/s-0036-1582397

43. Fasshauer M, Blüher M, Stumvoll M. Adipokines in Gestational Diabetes. Lancet Diabetes Endocrinol (2014) 2(6):488-99. doi: 10.1016/s2213-8587(13) 70176-1

44. Ye J, Zhu N, Sun R, Liao W, Fan S, Shi F, et al. Metformin Inhibits Chemokine Expression Through the AMPK/NF- $\mathrm{kb}$ Signaling Pathway. J Interferon Cytokine Res (2018) 38(9):363-9. doi: 10.1089/jir.2018.0061

45. Biden TJ, Boslem E, Chu KY, Sue N. Lipotoxic Endoplasmic Reticulum Stress, $\beta$ Cell Failure, and Type 2 Diabetes Mellitus. Trends Endocrinol Metab (2014) 25(8):389-98. doi: 10.1016/j.tem.2014.02.003

46. Barichello T, Simoes LR, Quevedo J, Zhang XY. Microglial Activation and Psychotic Disorders: Evidence From Pre-Clinical and Clinical Studies. Curr Top Behav Neurosci (2020) 44:161-205. doi: 10.1007/7854_2018_81

47. Rong C, Cui X, Chen J, Qian Y, Jia R, Hu Y. DNA Methylation Profiles in Placenta and its Association With Gestational Diabetes Mellitus. Exp Clin Endocrinol Diabetes (2015) 123(5):282-8. doi: 10.1055/s-0034-1398666

48. Simonova MA, Pivovarov VD, Ryazantsev DY, Dolgova AS, Berzhets VM, Zavriev SK, et al. Comparative Diagnostics of Allergy Using Quantitative Immuno-PCR and ELISA. Bioanalysis (2018) 10(10):757-67. doi: 10.4155/ bio-2017-0194

49. Tabatabaei MS, Islam R, Ahmed M. Applications of Gold Nanoparticles in ELISA, PCR, and Immuno-PCR Assays: A Review. Anal Chim Acta (2021) 1143:250-66. doi: 10.1016/j.aca.2020.08.030

50. Hirata Y, Katsukura Y, Henmi Y, Ozawa R, Shimazaki S, Kurosawa A, et al. Advanced Maternal Age Induces Fetal Growth Restriction Through Decreased Placental Inflammatory Cytokine Expression and Immune Cell Accumulation in Mice. J Reprod Dev (2021) 67(4):257-64. doi: 10.1262/ jrd.2021-034

51. Lawhorn C, Yuferov V, Randesi M, Ho A, Morgello S, Kreek MJ, et al. Genetic Diversity and Linkage Disequilibrium in the Chemokine Receptor CCR2CCR5 Region Among Individuals and Populations. Cytokine (2013) 64 (2):571-6. doi: 10.1016/j.cyto.2013.08.008

52. Silva-Carvalho WH, de Moura RR, Coelho AV, Crovella S, Guimarães RL. Frequency of the CCR5-Delta32 Allele in Brazilian Populations: A Systematic Literature Review and Meta-Analysis. Infect Genet Evol (2016) 43:101-7. doi: 10.1016/j.meegid.2016.05.024

53. Kuroda M, Sakaue H. Adipocyte Death and Chronic Inflammation in Obesity. J Med Invest (2017) 64(3.4):193-6. doi: 10.2152/jmi.64.193

54. Cheng D, Gong X, Wu Q, Yuan J, Lv Y, Yuan L, et al. High-Selectivity Fluorescent Reporter Toward Peroxynitrite in a Coexisting Nonalcoholic Fatty Liver and Drug-Induced Liver Diseases Model. Anal Chem (2020) 92 (16):11396-404. doi: 10.1021/acs.analchem.0c02277

55. Amatore C, Arbault S, Koh AC. Simultaneous Detection of Reactive Oxygen and Nitrogen Species Released by a Single Macrophage by Triple PotentialStep Chronoamperometry. Anal Chem (2010) 82(4):1411-9. doi: 10.1021/ ac902486x

56. Daniele G, Guardado Mendoza R, Winnier D, Fiorentino TV, Pengou Z, Cornell J, et al. The Inflammatory Status Score Including IL-6, TNF- $\alpha$, Osteopontin, Fractalkine, MCP-1 and Adiponectin Underlies Whole-Body Insulin Resistance and Hyperglycemia in Type 2 Diabetes Mellitus. Acta Diabetol (2014) 51(1):123-31. doi: 10.1007/s00592-013-0543-1

57. Chang TT, Chen JWJCD. Emerging Role of Chemokine CC Motif Ligand 4 Related Mechanisms in Diabetes Mellitus and Cardiovascular Disease: Friends or Foes? Cardiovasc Diabetol (2016) 15(1):117. doi: 10.1186/s12933-0160439-9

58. Barbé-Tuana FM, Klein D, Ichii H, Berman D, MCoffey L, Kenyon NS, et al. CD40-CD40 Ligand Interaction Activates Proinflammatory Pathways in Pancreatic Islets. Diabetes (2006) 55(9):2437-45. doi: 10.2337/db05-1673

59. Omatsu T, Cepinskas G, Clarson C, Patterson EK, Alharfi IM, Summers K, et al. CXCL1/CXCL8 (GRO Alpha/IL-8) in Human Diabetic Ketoacidosis Plasma Facilitates Leukocyte Recruitment to Cerebrovascular Endothelium In Vitro. Am J Physiol-Endocrinol Metab (2014) 306(9):E1077-84. doi: 10.1152/ ajpendo.00659.2013

60. Straczkowski M, Dzienis-Straczkowska S, Stêpieñ A, Kowalska I, Szelachowska M, Kinalska I. Plasma Interleukin-8 Concentrations Are Increased in Obese Subjects and Related to Fat Mass and Tumor Necrosis Factor-Alpha System. J Clin Endocrinol Metab (2002) 87(10):4602-6. doi: 10.1210/jc.2002-020135

61. Bruun JM, Lihn AS, Madan AK, Pedersen SB, Schiøtt KM, Fain JN, et al. Higher Production of IL-8 in Visceral vs. Subcutaneous Adipose Tissue. Implication of Nonadipose Cells in Adipose Tissue. Am J Physiol Endocrinol Metab (2004) 286(1):E8-13. doi: 10.1152/ajpendo.00269.2003

62. Tretjakovs P, Jurka A, Bormane I, Mackevics V, Mikelsone I, Balode L, et al. Relation of Inflammatory Chemokines to Insulin Resistance and Hypoadiponectinemia in Coronary Artery Disease Patients. Eur J Intern Med (2009) 20(7):712-7. doi: 10.1016/j.ejim.2009.08.004

63. Sajadi SMA, Khoramdelazad H, Hassanshahi G, Rafatpanah H, Hosseini J, Mahmoodi M, et al. Plasma Levels of CXCL1 (GRO-Alpha) and CXCL10 (IP10) are Elevated in Type 2 Diabetic Patients: Evidence for the Involvement of Inflammation and Angiogenesis/Angiostasis in This Disease State. Clin Lab (2013) 59(1-2):133-7. doi: 10.7754/Clin.Lab.2012.120225

64. Burke SJ, Karlstad MD, Eder AE, Regal KM, Lu D, Burk DH, et al. Pancreatic $\beta$-Cell Production of CXCR3 Ligands Precedes Diabetes Onset. Biofactors (2016) 42(6):703-15. doi: 10.1002/biof.1304

65. Tang Y, Zhou H, Chen A, Pittman RN, Field J. The Akt Proto-Oncogene Links Ras to Pak and Cell Survival Signals. J Biol Chem (2000) 275(13):9106-9. doi: $10.1074 /$ jbc.275.13.9106

66. Schulthess FT, Paroni F, Sauter NS, Shu L, Ribaux P, Haataja L, et al. CXCL10 Impairs $\beta$ Cell Function and Viability in Diabetes Through TLR4 Signaling. Cell Metab (2009) 9(2):125-39. doi: 10.1016/j.cmet.2009.01.003

67. Chen C, Jin X, Meng X, Zheng C, Shen Y, Wang Y. Inhibition of TnfoInduced Adhesion Molecule Expression by (Z)-(S)-9-Octadecenamide, N-(2 Hydroxyethyl,1-Methyl). Eur J Pharmacol (2011) 660(2-3):305-9. doi: 10.1016/j.ejphar.2011.04.009

68. Yin Q, Sun K, Xiang X, Juan J, Cao Y, Song J, et al. Identification of Novel CXCL12 Genetic Polymorphisms Associated With Type 2 Diabetes Mellitus: A Chinese Sib-Pair Study. Genet Test Mol Biomark (2019) 23(7):435-41. doi: $10.1089 / \mathrm{gtmb} .2018 .0149$

69. Al-Rikabi AHA, Tobin DJ, Riches-Suman K, Thornton MJ. Dermal Fibroblasts Cultured From Donors With Type 2 Diabetes Mellitus Retain an Epigenetic Memory Associated With Poor Wound Healing Responses. Sci Rep (2021) 11(1):1474. doi: 10.1038/s41598-020-80072-Z

70. Zhou F, Wang J, Wang K, Zhu X, Pang R, Li X, et al. Serum CXCL16 as a Novel Biomarker of Coronary Artery Disease in Type 2 Diabetes Mellitus: A Pilot Study. Ann Clin Lab Sci (2016) 46(2):184-9.

71. Zhao L, Wu F, Jin L, Lu T, Yang L, Pan X, et al. Serum CXCL16 as a Novel Marker of Renal Injury in Type 2 Diabetes Mellitus. PloS One (2014) 9(1): e87786. doi: 10.1371/journal.pone.0087786

72. van Lieshout AW, Popa C, Meyer-Wentrup F, Lemmers HL, Stalenhoef AF, Adema GJ, et al. Circulating CXCL16 Is Not Related to Circulating oxLDL in Patients With Rheumatoid Arthritis. Biochem Biophys Res Commun (2007) 355(2):392-7. doi: 10.1016/j.bbrc.2007.01.161

Conflict of Interest: The authors declare that the research was conducted in the absence of any commercial or financial relationships that could be construed as a potential conflict of interest.

Publisher's Note: All claims expressed in this article are solely those of the authors and do not necessarily represent those of their affiliated organizations, or those of the publisher, the editors and the reviewers. Any product that may be evaluated in 
this article, or claim that may be made by its manufacturer, is not guaranteed or endorsed by the publisher.

Copyright $\odot 2022$ Liu, Liu, Kaminga, McDonald, Wen and Pan. This is an openaccess article distributed under the terms of the Creative Commons Attribution
License (CC BY). The use, distribution or reproduction in other forums is permitted, provided the original author(s) and the copyright owner(s) are credited and that the original publication in this journal is cited, in accordance with accepted academic practice. No use, distribution or reproduction is permitted which does not comply with these terms. 